\title{
New or Unknown Interferent
}

National Cancer Institute

\section{Source}

National Cancer Institute. New or Unknown Interferent. NCI Thesaurus. Code C139523.

New or unknown endogenous or exogenous interferent (sample) identified. 